\title{
因HAD
}

ISSN-L: 2530-5115

(c) (1)(2)

BY NO SA

DOI: http://doi.org/10.22585/hospdomic.v3i4.81

\section{Rotación a Metadona en domicilio como alternativa en dolor refractario oncológico}

Rotation of Methadone in address as an alternative in oncological refractory pain

Aarón Gutiérrez Pastor', Annabella Freitas Amorós', Rafael Lopez-Bas'

1. Hospital San Juan de Alicante. Unidad de Hospitalización a Domicilio. Sant Joan d'Alacant, España.

Correspondencia/Correspondence

Aarón Gutiérrez Pastor

Recibido/Received

27.06.2019

Aceptado/Accepted

01.09 .2019
Conflicto de Intereses/Competing interest Los autores y autora del trabajo declaran no tener ningún conflicto de interés en dolor refractario oncológico. Hosp Domic. 2019;3(4):279-88 


\section{RESUMEN}

Los opioides son la piedra fundamental en el tratamiento farmacológico del dolor oncológico, llegando a controlar al $80 \%$ de los casos; permaneciendo un $20 \%$ en que se mantiene un dolor refractario, por no conseguir aliviar el dolor a pesar de un incremento suficiente en la dosis y/o por aparición de efectos secundarios indeseables.

Una de las medidas propuestas para el manejo del dolor refractario consiste en la Rotación de Opioides, cuyo objetivo es obtener un equilibrio entre control analgésico y efectos secundarios. Es aquí donde la Metadona parece jugar un importante papel como medicamento a utilizar como segunda línea para el control sintomático. Sus características farmacocinéticas: gran biodisponibilidad oral, su actividad agonista en receptores opioides, junto al efecto antagonista sobre los receptores $\mathrm{N}$-metil-D-Aspartato (NMDA), la convierten en una buena alternativa para conseguir el control del dolor refractario que habitualmente tiene gran componente neuropático. Sin embargo, debido a una vida media larga e impredecible, requiere ser realizada por equipos con experiencia.

Palabras clave: Dolor oncológico; Analgésicos opioides; Metadona; Servicios de Atención a Domicilio Provisto por Hospital.

\section{ABSTRACT}

Opioids are the cornerstone in the pharmacological treatment of oncological pain, reaching $80 \%$ control of cases; remaining $20 \%$ in which a refractory pain is maintained, because of not managing to alleviate the pain despite a sufficient increase in the dose and / or by the appearance of undesirable side effects.

One of the measures proposed for the management of refractory pain consists of the rotation of opioids, whose objective is to obtain a balance between analgesic control and side effects. This is where Methadone seems to play an important role as a drug to be used as a second line for symptomatic control. Its pharmacokinetic characteristics: great oral bioavailability, its agonist activity in opioid receptors, together with the antagonistic effect on N-methyl-D-Aspartate (NMDA) receptors, make it a good alternative to achieve control of refractory pain that usually has a large neuropathic component. However, due to a long and unpredictable half-life, it needs to be done by experienced teams.

Palabras clave: Cancer pain; Analgesics opioid; Methadone; Home Care Services, Hospital-Based. 


\section{INTRODUCCIÓN}

Según las recomendaciones de la Sociedad Española de Cuidados Paliativos el dolor producido por el cáncer es, posiblemente, uno de los síntomas más frecuentes, así como el que más temor genera en los pacientes con un diagnóstico oncológico (1). Éste llega a aparecer hasta en el 30\% de los mismos en el momento del diagnóstico inicial de la enfermedad, pudiendo alcanzar hasta en el $70-80 \%$ de los pacientes en los momentos finales de su vida.

El dolor es uno de los síntomas más comunes en los enfermos con un diagnóstico de cáncer, por su alta prevalencia y severidad. Además, el Observatorio Europeo del Cáncer (ECO-OEC) estima que la incidencia de cáncer presenta una tendencia en aumento; alcanzando cifras en 2018 de 683,5 casos por cada 100.000 habitantes y suponiendo la muerte de más de 294,3 por cada 100.000 pacientes (2). Durante toda la historia natural del cáncer, entre el 50\% de los pacientes presentan dolor, alcanzando al $75 \%$ en situación de enfermedad avanzada y terminal (3). Además, del total de estos enfermos, en torno a un $25-30 \%$ presentarán un dolor de alta severidad, mientras que un $60 \%$ sufrirá más de un tipo de dolor y un 30\% padecerá 3 o más tipos diferentes de dolor (4). Por otro lado, casi un 30\% de los enfermos oncológicos no reciben un tratamiento analgésico adecuado debido probablemente a la dificultad intrínseca del propio dolor y a un adiestramiento parcial y deficitario de los profesionales sanitarios en el uso de opioides potentes (5).

Existe escasa literatura acerca de estudios epidemiológicos relacionados sobre registros de dolor por cáncer en países industrializados. Además, existe un subregistro del dolor relacionado con cáncer (6). Las razones que explicarían este fenómeno son complejas y no se entienden bien. Aparentemente, se debe a una serie de creencias por parte de los pacientes, familias y profesionales de la salud. Las razones esgrimidas por The British Pain Society's, incluyen los siguientes argumentos (6):

- Creencia de que el dolor es inevitable en el cáncer.

- Creencia de que los "buenos" pacientes no se quejan de dolor.

- Preocupación de que hablar sobre el dolor pueda distraer al médico del tratamiento del cáncer.

- Temor a la adicción al medicamento.

- Inquietudes acerca de la tolerancia.

- Inquietudes acerca de los efectos secundarios.

- Miedo a que el dolor signifique progresión de enfermedad.

El conocimiento de la prevalencia y del tratamiento integral del dolor por cáncer permitiría optimizar claramente la atención, mejorando la disponibilidad de los medicamentos opioides, así como implementar políticas de educación en dolor, con una correcta asignación de recursos económicos, principalmente en el campo de la investigación (7).

Los opioides constituyen la principal herramienta en la lucha contra el dolor en el cáncer. Éstos no tienen techo analgésico y las dosis requeridas varían considerablemente de unos pacientes a otros. Siguiendo las recomendaciones de la Organización Mundial de la Salud (OMS), se consigue un buen control analgésico en más del $80 \%$ de los casos, permaneciendo un $20 \%$ de los mismos en que se mantiene un dolor refractario, bien por no conseguir aliviar el dolor a pesar de un incremento suficiente en la dosis del opioide, o bien por la aparición de efectos secundarios indeseables que dificultan el manejo del paciente. Una de las medidas propuestas para el manejo del dolor refractario, pasa por la realización de una rotación de opioides, técnica que consiste en la sustitución del opioide previo por otro con el objetivo de obtener un equilibrio entre control analgésico y los efectos secundarios. Es aquí donde la Metadona parece jugar un importante papel como medicamento a utilizar en segunda línea para el control sintomático de estos pacientes. Las características far- 
macocinéticas de la metadona por su gran biodisponibilidad oral y su mecanismo de acción dual como agonista en receptores opioides, y por su efecto antagonista sobre los receptores NMDA, lo que disminuye el desarrollo de tolerancia a los opioides. Todo ello, la convierten en una muy buena alternativa para conseguir el control del dolor refractario, que habitualmente tiene un gran componente neuropático. Por otro lado, y debido a presentar una vida media larga e impredecible, requiere que la rotación sea realizada por equipos con amplia experiencia principalmente en ámbito extrahospitalario.

\section{DESARROLLO DE LA EXPERIENCIA}

Presentamos el caso de un paciente de 55 años, ingresado a cargo de nuestra Unidad de Hospitalización a Domicilio (UHD) para control de síntomas, con diagnóstico de adenocarcinoma de próstata T4N1M1c en estadio IV Gleason 4+4, con valores de Antígeno prostático específico (PSA) de 110,47ng/mL y fosfatasa alcalina (FA) de $142 \mathrm{U} / \mathrm{L}$ iniciales, y metástasis óseas múltiples: en columna vertebral dorsal y lumbar, parrillas costales, escápula izquierda, esternón, sacro, pelvis y vertiente proximal de ambos fémures. Así mismo presentaba varios focos metastásicos a nivel hepático, mediastínico, retroperitorneal y peritoneal (tabla 1); no susceptible de más tratamiento quimioterápico con intención curativa por el servicio de oncología (figuras 1-3).

Había recibido radioterapia antiálgica sobre pelvis, caderas y región lumbar por las lesiones metastásicas óseas a dosis submáximas (20Gy) 4 meses antes. Desde su ingreso, presenta un difícil manejo del control del dolor, a pesar de haber recibido tratamiento inicialmente con parches de Fentanilo TTS a dosis de 300mcg/hr incluso cada 48 horas; Dexametasona a dosis de $12 \mathrm{mg} / \mathrm{día}$ (8-4-0); y Pregabalina 150mg/12 horas.

Se intenta realizar rotación de opioides con infusor subcutáneo de morfina a dosis de $360 \mathrm{mg} / \mathrm{día}$ junto a rescates de Fentanilo de liberación rápida de $400 \mathrm{mcg} /$ pulsación (llegando a requerir unos 5 rescates por día). Tras 72 horas con esta dosis, no se consigue controlar el dolor y comienza a presentar cuadro compatible con Neurotoxidad Inducida por Opiáceos (NIO): mioclonías, bradipsiquia y delirium. Se decide ante fenómeno de hiperalgesia, realizar rotación gradual en 3 días a Metadona, siguiendo esquema de Ripamonti (tabla 2 y tabla 3 ). 
Tabla 1. Sistema TNM de estadificación del cáncer de próstata (11)

\begin{tabular}{|c|c|c|}
\hline Etapa & $\begin{array}{l}\text { Agrupamiento para } \\
\text { establecer la etapa }\end{array}$ & Definiciones \\
\hline \multirow[t]{3}{*}{1} & $\begin{array}{l}\text { cT1, N0, M0 } \\
\text { Grupo de Grado } 1 \\
\text { PSA }<10\end{array}$ & \multirow{13}{*}{$\begin{array}{l}\text { T: Extensión del tumor principal } \\
\text { - T1 y T2: Tumor localizado en la próstata } \\
\text { - cT1: El tumor no se puede palpar con tacto rectal o } \\
\text { verse con un estudio por imagen. } \\
\text { - cT2a: El tumor se puede palpar con tacto rectal o } \\
\text { verse con un estudio por imagen. Se encuentra en } \\
\text { una mitad o menos de un lado de la próstata. } \\
\text { - pT2a: La próstata ha sido removida con cirugía y el } \\
\text { tumor se encuentra localizado en la misma. } \\
\text { - cT2b: El tumor se puede palpar con tacto rectal o } \\
\text { verse con un estudio por imagen. Se encuentra en } \\
\text { más de la mitad de un lado de la próstata. } \\
\text { - cT2c: El tumor se puede palpar con tacto rectal o } \\
\text { verse con un estudio por imagen. Se encuentra en } \\
\text { ambos lados de la próstata. } \\
\text { - T3: El cáncer se ha extendido a las vesículas seminales. } \\
\text { - T4: El cáncer se ha extendido al esfínter uretral, recto, } \\
\text { vejiga y/o a la pared de la pelvis } \\
\text { N: Afectación de ganglios linfáticos }\end{array}$} \\
\hline & $\begin{array}{l}\text { cT2a, N0, M0 } \\
\text { Grupo de Grado } 1 \\
\text { PSA }<10\end{array}$ & \\
\hline & $\begin{array}{l}\text { pT2, N0, M0 } \\
\text { Grupo de Grado } 1 \\
\text { PSA }<10\end{array}$ & \\
\hline \multirow[t]{3}{*}{ II } & $\begin{array}{l}\text { cT1, No, M0 } \\
\text { Grupo de Grado } 1 \\
\text { PSA } 10-20\end{array}$ & \\
\hline & $\begin{array}{l}\text { cT2a o pT2, NO, M0 } \\
\text { Grupo de Grado } 1 \\
\text { PSA } 10-20\end{array}$ & \\
\hline & $\begin{array}{l}\text { cT2b o cT2c, N0, M0 } \\
\text { Grupo de Grado } 1 \\
\text { PSA }<20\end{array}$ & \\
\hline IIB & $\begin{array}{l}\text { T1 o T2, N0, M0 } \\
\text { Grupo de Grado } 2 \\
\text { PSA }<20\end{array}$ & \\
\hline IIC & $\begin{array}{l}\text { T1 o T2, N0, M0 } \\
\text { Grupo de Grado } 3 \text { ó } 4 \\
\text { PSA }<20\end{array}$ & \\
\hline IIIA & $\begin{array}{l}\text { T1 o T2, N0, M0 } \\
\text { Grupo de Grado } 1 \text { a } 4 \\
\text { PSA } \geq 20\end{array}$ & \\
\hline IIIB & $\begin{array}{l}\text { T3 o T4, N0, M0 } \\
\text { Grupo de Grado } 1 \text { a } 4 \\
\text { Cualquier PSA }\end{array}$ & \\
\hline IIIC & $\begin{array}{l}\text { Cualquier T, NO, MO } \\
\text { Grupo de Grado } 5 \\
\text { Cualquier PSA }\end{array}$ & \\
\hline IVA & $\begin{array}{l}\text { Cualquier T, N1, M0 } \\
\text { Cualquier Grupo de Grado } \\
\text { Cualquier PSA }\end{array}$ & \\
\hline IVB & $\begin{array}{l}\text { Cualquier T, cualquier N, M1 } \\
\text { Cualquier Grupo de Grado } \\
\text { Cualquier PSA }\end{array}$ & \\
\hline
\end{tabular}


Notas Clínicas: Gutiérrez Pastor et al

Figura 1. Conglomerados adenopáticos interaortocava y paraórticos

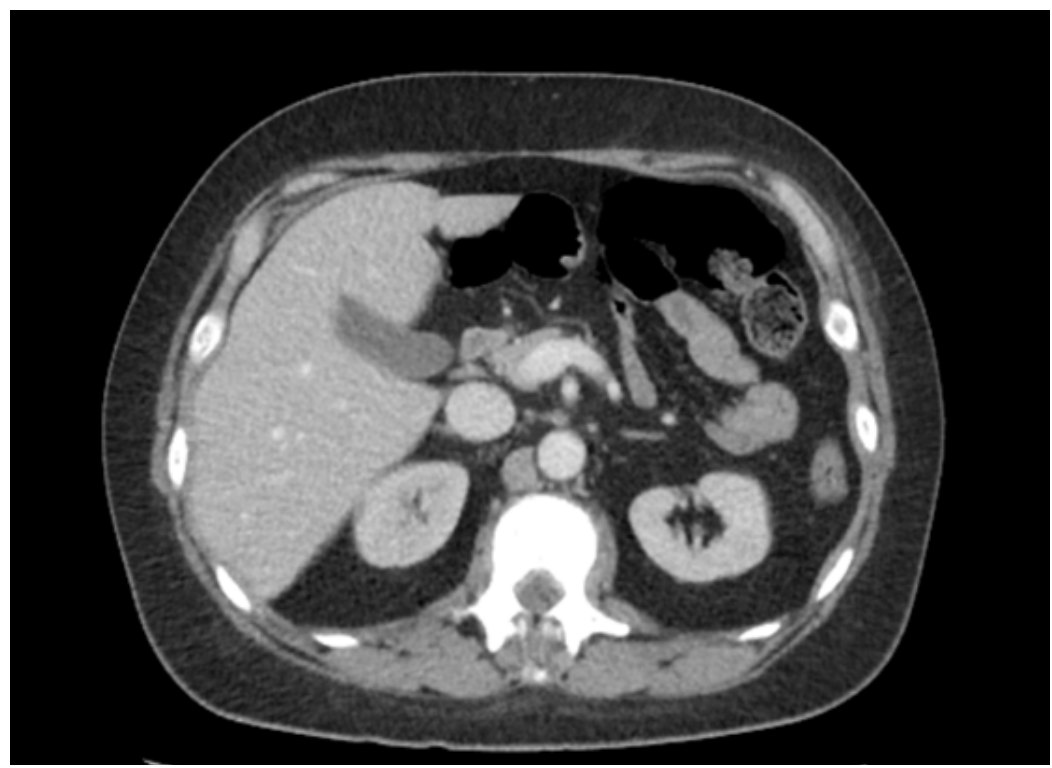

Figura 2. Metástasis blásticas con componente de tejido de partes blandas asociado e infiltración de músculo iliaco y glúteo menor derechos

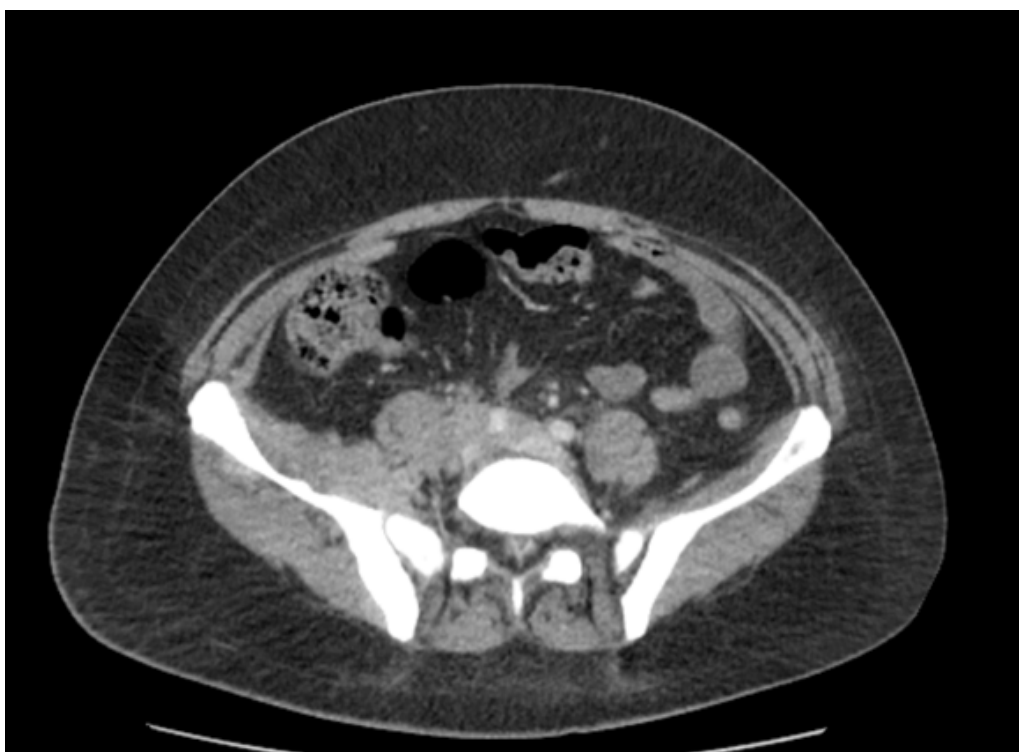


Rotación a Metadona en domicilio como alternativa en dolor refractario oncológico

Figura 3. Gammagrafía ósea Cuerpo entero

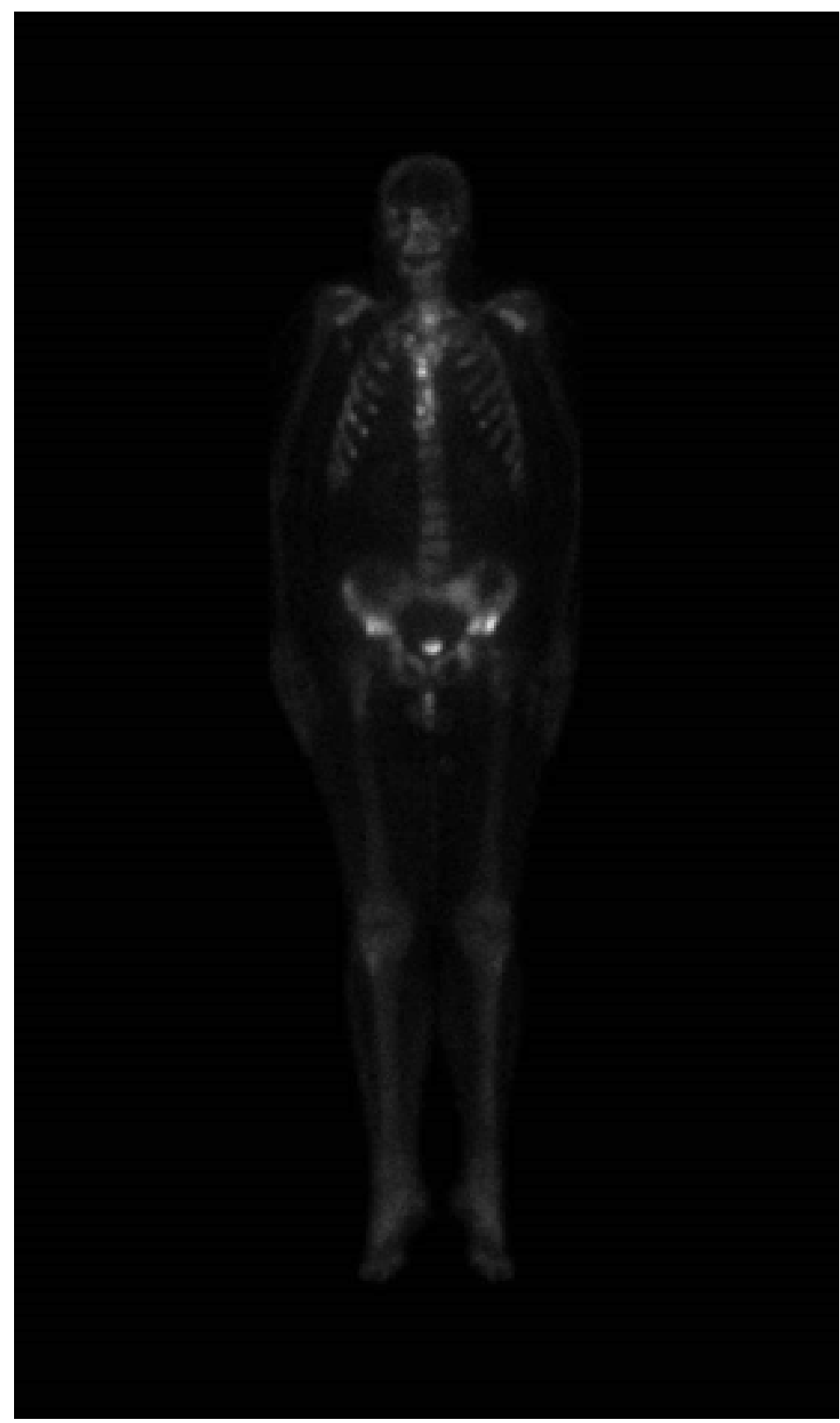


Tabla 2. Ratios equi-analgésicos morfina-metadona de acuerdo a dosis previas de morfina oral (8)

\begin{tabular}{|l|l|}
\hline Dosis previa de Morfina en $\mathbf{2 4}$ horas & Ratio recomendado Morfina oral: Metadona oral* \\
\hline Menos de $90 \mathrm{mg}$ & $4: 1$ \\
\hline 90-300 mg & $8: 1$ \\
\hline Mayor de $300 \mathrm{mg}$ & $12: 1$ \\
\hline
\end{tabular}

Tabla 3. Método de rotación gradual a metadona en 3 días (8)

\begin{tabular}{|l|l|l|}
\hline \multicolumn{2}{|c|}{ Dosis Morfina equivalente $>100$ mg* } & Dosis de Metadona** \\
\hline Día 1 & Reducción de 30-50\% & Reemplazar opioides usando ratio Morfina-Metadona 10:1 \\
\hline Día 2 & $\begin{array}{l}\text { Reducción adicional } \\
30-50 \% \text { de la dosis }\end{array}$ & $\begin{array}{l}\text { Aumentar Metadona si hay dolor moderado a severo. (Dolor } \\
\text { transitorio manejado con opioides de rescate) }\end{array}$ \\
\hline Día 3 & $\begin{array}{l}\text { Discontinuar } \\
\text { (Diaria de Metadona cada ocho horas. Rescate: 10\% de la dosis }\end{array}$ \\
\hline $\begin{array}{l}\text { * Dosis Morfina equivalente menor a 100mg: suspensión inmediata de Morfina y administrar Metadona 5mg } \\
\text { ** La dosis total de Metadona se da dividida en tres tomas diarias (cada ocho horas) }\end{array}$ \\
\hline
\end{tabular}

Se inicia Metadona a dosis de 20 mg/8horas, reduciendo cada día en torno a 1/3 de la dosis de morfina que llevaba en el infusor (primer día 250 mg, segundo día 150 mg y al tercer día se retiró el infusor subcutáneo). Desde las primeras 24 horas, el paciente describe mejoría clínica, pasando a tener en la Escala Visual Analógica (EVA) una puntuación de 10/10 hasta alcanzar un EVA de 3/10 (70\% de mejoría de la percepción del dolor) a los 5 días. No presentó efectos secundarios y permaneció un total de 52 días con Metadona oral; disminuyendo, incluso, los fármacos coadyuvantes y consiguiendo una adecuada calidad de vida del paciente, pudiendo descansar y deambular por domicilio.

Sin embargo, se produce la progresión de enfermedad con infiltración del canal medular. Se inició radioterapia urgente y se inició corticoterapia a altas dosis. A pesar de ello, presenta empeoramiento continuo tras lo que presenta una parada cardiorrespiratoria y fallecimiento del paciente. 


\section{CONCLUSIONES}

El dolor es un síntoma de alta prevalencia en los pacientes con diagnóstico de cáncer, haciendo que disminuya la actividad del enfermo, interfiriendo con el apetito, el sueño, el estado del ánimo y conllevando a la pérdida del autocontrol. Además, a pesar de seguir las recomendaciones de la denominada Escalera analgésica de la OMS, existe un grupo de pacientes, hasta un $30 \%$, en quienes se plantea la necesidad de realizar una rotación de opioides, bien por una pobre respuesta analgésica, por aparición de toxicidad, o una combinación de ambas (9). Esta rotación suele ser eficaz con una mejoría en el control del dolor en el 40-80\% de los casos.

El dolor en pacientes oncológicos suele tener mucho componente neuropático; de ahí que la Metadona sea considerada como el analgésico de $2^{\mathrm{a}}$ línea para realizar una rotación de opioides por su mecanismo dual $(10,11)$. Como en el estudio prospectivo de Porta-Sales et al. (11), con este caso se demuestra la eficacia y seguridad de la rotación escalonada a metadona en pacientes oncológicos en ámbito domiciliario con mal control del dolor, siempre y cuando ésta se realice por equipos adiestrados en el manejo de este medicamento.

\section{BIBLIOGRAFÍA}

1. Sociedad Española de Cuidados Paliativos (SECPAL). Recomendaciones de la Sociedad Española de Cuidados Paliativos (SECPAL). Madrid, España: Ministerio de Sanidad y Consumo; 1993.

2. ECO/OEC. European Cancer Observatory, Observatoire Européen du Cancer. International Agency for Research on Cancer, Lyon, 2009 [citado 1 jun 2019]. Disponible en: http://eu-cancer.iarc.fr.

3. Hui D, Bruera E. A personalized approach to assessing and managing pain in patients with cancer. J Clin Oncol. 2014 Jun 1;32(16):1640-6. DOI: 10.1200/JCO.2013.52.2508; PMID: 24799495

4. Grond S, Zech D, Diefenbach C, Radbruch L, Lehmann KA. Assessment of cancer pain: A prospective evaluation in 2266 cancer patients referred to a pain service. Pain. 1996;64(1):107-14. DOI: 10.1016/0304-3959(95)00076-3; PMID:8867252.

5. Cleeland CS, Gonin R, Hatfield AK, Edmonson JH, Blum RH, Stewart JA, et al. Pain and its treatment in outpatients with metastatic cancer. N Engl J Med. 1994 Mar 3; 330(9):592-6. DOI: 10.1056/NEJM199403033300902; PMID: 7508092

6. The British Pain Society. Cancer Pain Management [monografía en Internet]. London, United Kingdom: The British Pain Society; 2010 [citado 25 ago 2019]. Disponible en: http://bit. Iy/2ZqNMTE

7. Franks PJ, Salisbury C, Bosanquet N, Wilkinson EK, Lorentzon M, Kite S, et al. The level of need for palliative care: A systematic review of the literature. Palliat Med. 2000;14(2):93-104. DOI: 10.1191/026921600669997774; PMID: 10829143

8. Patricia Papa M. Modalidades de rotación morfina a metadona en pacientes con dolor oncológico. Rev Med Urug. 2009; 25:124-130.

9. Quigley C. Opioid switching to improve pain relief and drug tolerability. Cochrane Database Syst Rev. 2004;(3):CD004847. DOI: 10.1002/14651858.CD004847.pub2; PMID: 15266542 
Notas Clínicas: Gutiérrez Pastor et al

10. Mercadante S, Bruera E. Opioid switching: a systematic and critical review. Cancer Treat Rev. 2006;32(4):304-15. DOI: 10.1016/j.ctrv.2006.03.001; PMID: 16624490

11. Porta-Sales J, Garzón-Rodríguez C, Villavicencio-Chávez C, Llorens-Torromé S, González-Barboteo J. Efficacy and Safety of Methadone as a Second-Line Opioid for Cancer Pain in an Outpatient Clinic: A Prospective Open-Label Study. Oncologist. 2016;21(8):981-7. DOI: 10.1634/ theoncologist.2015-0503; PMID: 27306912

12. American Joint Committee on Cancer. Prostate. In: AJCC Cancer Staging Manual. 8th ed. New York, NY: Springer; 2017: 715-725. 